\title{
RETROSPECTIVA DA PRODUÇÃO CIENTÍFICA DO SIBRACEn: (1988-1994)*
}

\author{
Maria Márcia Bachion** \\ Emilia Campos de Carvalho*** \\ Rosamary A. Garcia Stuchi****
}

BACHION, M.M.; CARVALHO, E.C.de; STUCHI, R.A.G. Retrospectiva da produção científica do SIBRACEn: (1988-1994). Rev.latino-am.enfermagem, Ribeirão Preto, v. 6, n. 2, p. 47-55, abril 1998.

Buscou-se identificar algumas características da produção científica acerca da Comunicação em Enfermagem tendo-se como amostra os trabalhos de temas livres apresentados nos quatro eventos - Simpósio Brasileiro de Comunicação em Enfermagem (1988, 1990, 1992 e 1994). Verificou-se que a maioria dos autores é composta por docentes, que realizam as investigações preferencialmente em grupos, enfocando principalmente como sujeitos as diades (profissionais e clientela), estabelecendo como objeto de estudo aspectos da interação (qualidade e efetividade), bem como aspectos da produção/utilização do conhecimento em Enfermagem. O contexto interpessoal é o usualmente mais pesquisado. A área mais estudada é a assistencial e o ambiente é o hospitalar. As investigações são na maioria do tipo não experimento. Os resultados arrolados pelos seus autores evidenciam aspectos de inadequação da comunicação nas áreas da assistência (incluindo a administração), da educação (principalmente da clientela) e da pesquisa. Ao mesmo tempo percebe-se a tendência subjacente de busca de soluções inovadoras, bem como avanço no conhecimento do processo de comunicação.

UNITERMOS: comunicação em enfermagem, produção científica

\section{INTRODUÇÃO}

A investigação em Enfermagem no Brasil, cujo início é recente, conta com diversos estudos que retratam a produção nesta área. Os anais de eventos nacionais como o Seminário Nacional sobre Ensino de Pós-Graduação e Pesquisa em Enfermagem, realizado em 1982 e os Seminários Nacionais de Pesquisa em Enfermagem (SENPE), realizados a partir de 1979 constituem fontes de pesquisas sobre a produção e divulgação do conhecimento, evidenciando vazios no mesmo.

Uma da áreas carentes de investigações em nosso país, conforme se comprova pelos estudos de ENOKI et al.(1987); AGUIAR et al.(1991) e CARVALHO (1989, 1990), tem sido a comunicação em enfermagem. Estes autores apresentaram, respectivamente, investigações acerca da produção científica sobre comunicação em enfermagem nos períodos de 1975 a 1985 e 1985 a 1990, em âmbito nacional, bem como nos períodos de 1975 a 1985 e 1985 a 1989, em âmbito internacional.
CARVALHO(1989) menciona que no período de 1975 a 1985 apenas trinta e três publicações, ou seja, $1,9 \%$ dos artigos indexados nos INI***** e CINAHL $* * * * * *$, referiam-se a autores nacionais. Em estudo no mesmo período, ENOKI et al.(1987) identificaram, em 5 periódicos nacionais, 30 artigos. Cumpre destacar que a discreta divergência dos dados é atribuida aos periódicos indexados, que não constituem a mesma amostra, já que os critérios de indexação dos periódicos são seletivos. Estes dados refletem a baixa freqüência de estudos nesta temática, nos periódicos nacionais.

Embora não indexados, os Anais dos 3 primeiros Simpósios Brasileiro de Comunicação em Enfermagem $* * * * * * *$, congregam o maior número de estudos específicos sobre este tema na Enfermagem, configurando-se em importante acervo para pesquisas bibliográficas. Cumpre destacar que não foi adotada a estratégia de divulgação dos Anais dos dois últimos eventos.

\footnotetext{
* Trabalho apresentado no $5^{\circ}$ Simpósio Brasileiro de Comunicação em Enfermagem - Ribeirão Preto, maio, 1996

** Professor Titular junto ao Departamento de Enfermagem - Universidade Federal de Goiás

*** Professor Titular junto à Escola de Enfermagem de Ribeirão Preto - Universidade de São Paulo

**** Mestranda da Escola de Enfermagem de Ribeirão Preto - Área Enfermagem Fundamental - Enfermeira do HCFMRP

$* * * * *$ INI - International Nursing Index

$* * * * * *$ Cumulative Index to Nursing \& Allied Health Literature

******* Evento realizado bienalmente pelo Departamento de Enfermagem Geral e Especializada da Escola de Enfermagem de Ribeirão Preto da Universidade de São Paulo
} 
Concordando com POLIT \& HUNGLER(1991)

que o conhecimento é acumulado através da condução e avaliação de muitos estudos dirigidos às mesmas ou similares questões de pesquisa, para o presente trabalho, exploraremos os dados divulgados como temas livres e apresentados nos eventos. Acreditamos ser este volume uma mostra representativa, como fonte de dados, para o alcance dos seguintes objetivos:

- identificar: a área de formação, atuação e número de autores; o(s) sujeito(s) e objeto(s) dos estudos; o contexto de comunicação pesquisado; o tipo de pesquisa; os referenciais teóricos utilizados;

- identificar e agrupar os resultados destas pesquisas por áreas (Assistência, Educação, Pesquisa) e reconhecer o ambiente onde se desenvolvem;

- descrever, em termos gerais, os achados destes estudos quanto à comunicação na área da assistência, da educação e da pesquisa.

\section{PRODEDIMENTO METODOLÓGICO}

Para análise das informações sobre os trabalhos de "temas livres" apresentados nos quatro simpósios, foi elaborado um instrumento de coleta de dados em forma de fichas, cujos itens foram, em parte inspirados no formulário usado por CARVALHO(1989), compondo o seguinte corpo de elementos a serem registrados:

- número do artigo (ordem de publicação)

- título do trabalho

- número de autores, área de formação (enfermeiro/ não enfermeiro) e atuação (aluno: de graduação em enfermagem, pós-graduação sensu lato, sensu strictu, em iniciação científica, em aperfeiçoamento; docente; enfermeiro assistencial - incluindo aqueles que estão em cargos administrativos em instituições prestadoras de cuidados à saúde; outros). Consideramos neste item exclusivamente as informações registradas nos artigos.

- sujeito(s) do trabalho

- objeto

- contexto de comunicação abordado - (intrapessoal, interpessoal, organizacional, grupal e de massa). Adotamos para esta classificação as proposições de LITTLEJOHN (1982)

- área de investigação (assistência; educação - do alunado, do paciente, equipe, comunidade; administração; pesquisa; outros)

- ambiente da pesquisa (hospital, ambulatório, unidade básica de saúde, escolas, centros comunitários, residências, outros).

- tipo de pesquisa: consideramos neste tópico as definições de POLIT \& HUNGLER(1991) que consideram: experimento, quase experimento e pesquisa não experimental.

- referencial teórico na área de comunicação consideraremos exclusivamente que foi explicitado pelos (s) autores(s).

\section{APRESENTAÇÃO E ANÁLISE DOS RESULTADOS}

Os resultados serão apresentados na ordem adotada para o procedimento metodológico. Foram analisadas 154 publicações (Tabela 1) sendo as maiores freqüências as dos dois últimos eventos, sugerindo a consolidação do mesmo como veículo de divulgação da produção científica deste tema.

Ao se examinar o número de autores por publicação, nos 4 eventos, pode-se observar que a maioria foi escrita por 2 pessoas (36,4\%), seguindo-se 3 autores $(20,8 \%)$, com frequência semelhante aos escritos por um autor $(19,5 \%)$. Os trabalhos escritos por 4 ou mais autores são menos frequentes.

Tabela 1 - Freqüência de temas livres apresentados em cada evento

\begin{tabular}{c|c|c|c|c|c|c|c|c}
\hline \multirow{2}{*}{ ANO } & \multirow{2}{*}{ N$^{\circ}$ de Publicação } & \multicolumn{7}{|c}{ N $^{\circ}$ de Autores/Trabalhos } \\
\cline { 3 - 10 } & & 1 & 2 & 3 & 4 & 5 & 6 & 7 \\
\hline 1988 & 24 & 4 & 8 & 5 & 2 & 3 & 1 & 1 \\
\hline 1990 & 38 & 10 & 9 & 12 & 4 & 3 & - & - \\
\hline 1992 & 51 & 9 & 27 & 6 & 9 & - & - & - \\
\hline 1994 & 41 & 7 & 12 & 9 & 6 & 5 & 2 & - \\
\hline TOTAL N $^{\circ}$ & $\mathbf{1 5 4}$ & $\mathbf{3 0}$ & $\mathbf{5 6}$ & $\mathbf{3 2}$ & $\mathbf{2 1}$ & $\mathbf{1 1}$ & $\mathbf{3}$ & $\mathbf{1}$ \\
\hline \% & $\mathbf{1 0 0}$ & $\mathbf{1 9 5}$ & $\mathbf{3 6 , 4}$ & $\mathbf{2 0 , 8}$ & $\mathbf{1 3 , 6}$ & $\mathbf{7 , 1}$ & $\mathbf{1 , 9}$ & $\mathbf{0 , 6}$ \\
\hline
\end{tabular}

Do ponto de vista da difusão do conhecimento, tal achado é animador pois a realização de pesquisa em grupos favorece o conhecimento dos resultados por um maior número de pessoas.

Quanto a área de formação dos autores, notase, pela Tabela 2, predomínio de enfermeiros $(82,2 \%)$; discreta interdisciplinaridade na produção do conhecimento pode ser observada pela participação de autores não enfermeiros (7,7\%); a participação de alunos de graduação $(10,1 \%)$ reflete o envolvimento destes em atividades de iniciação científica. 
Tabela 2 - Freqüência da área de formação e atuação dos autores

\begin{tabular}{|c|c|c|c|c|c|c|c|c|c|c|}
\hline \multirow[t]{2}{*}{ ANO } & \multicolumn{4}{|c|}{ AREA DE FORMAÇÃO } & \multicolumn{6}{|c|}{ AREA DE ATUAÇÃO } \\
\hline & Enfermeiro & $\begin{array}{c}\text { Não } \\
\text { Enfermeiro }\end{array}$ & $\begin{array}{c}\text { Ahuno } \\
\text { Graduação }\end{array}$ & Total & Docente & $\begin{array}{c}\text { Ahuno } \\
\text { Graduação }\end{array}$ & $\begin{array}{l}\text { Enfermeiro } \\
\text { Assistencial }\end{array}$ & $\begin{array}{l}\text { Ahuno } \\
\text { Pós-Grad. }\end{array}$ & Outro & Total \\
\hline 1988 & 55 & 6 & 14 & 75 & 45 & 14 & 4 & 11 & 1 & 75 \\
\hline 1990 & 77 & 9 & 9 & 95 & 60 & 9 & 14 & 10 & 2 & 95 \\
\hline 1992 & 98 & 6 & 12 & 116 & 81 & 12 & 13 & 9 & 1 & 116 \\
\hline 1994 & 103 & 10 & 6 & 119 & 67 & 7 & 23 & 18 & 4 & 119 \\
\hline Total $N^{w}$ & 333 & 31 & 41 & 405 & 253 & 42 & 54 & 48 & 8 & 405 \\
\hline$\%$ & 82,2 & 7,7 & 10,1 & 100 & 62,5 & 10,4 & 13,3 & 11,8 & 2,0 & 100 \\
\hline
\end{tabular}

Inclui-se na categoria ENFERMEIRO os docentes enfermeiros, alunos de pós-graduação, bolsistas de aperfeiçoamento e enfermeiros assistenciais, melhor visualizado pela área de atuação. Nesta variável temos que destacar que foi considerada a menção feita pelo próprio autor, isto é, se por exemplo um autor for aluno de pós-graduação e docente em outra instituição consideramos a primeira menção feita pelo autor.

A participação do docente como autor é predominante $(62,5 \%)$; contudo pode se observar que o mesmo docente apresentou, em um mesmo evento, mais de uma produção em conjunto com outros docentes ou seus orientandos de pós-graduação ou iniciação científica, refletindo a participação de grupos de pesquisa.

Já foi evidenciado por POLIT \& HUNGLER(1991) a importância da realização e utilização de pesquisas ao desenvolvimento da profissão. A pesquisa deve fazer parte do cotidiano profissional e pode envolver tanto o enfermeiro assistencial como o aluno de graduação. Neste sentido a participação destes nos grupos de pesquisa ou realizações independentes devem ser sempre estimuladas. Em nosso estudo o enfermeiro assistencial (que em alguns casos é ex-aluno de pós-graduação ou bolsista de aperfeiçoamento) contribuiu discretamente como autor $(13,3 \%)$. Neste último caso, cumpre relembrar que vários pós-graduandos foram incluídos na categoria docente. Devem ser estimulados, cada vez mais, para ampliar esta aproximação pesquisa-assistência - ensino.

Quanto aos sujeitos das publicações os dados foram agrupados segundo a menção dos seus próprios autores e constituiram a Tabela 3 .

Tabela 3 - Freqüência dos tipos de sujeitos pesquisados

\begin{tabular}{|c|c|c|c|c|c|c|c|}
\hline \multicolumn{2}{|r|}{ SUJEITOS } & 1988 & 1990 & 1092 & 1994 & \multicolumn{2}{|c|}{ TOTAL } \\
\hline \multirow{8}{*}{ Díades } & Equipe Saúde x Pacientes & & 2 & 2 & 1 & 5 & 3,2 \\
\hline & Equipe Enf. x Pacientes/Fatniliares & 1 & 2 & 8 & - & 11 & 7,1 \\
\hline & Equipe Enf. $x$ Alunos Enfermagem & & & 1 & 1 & 2 & 1,3 \\
\hline & Enfermeiro $\mathrm{x}$ Aluno & & & 1 & & 1 & 0,6 \\
\hline & Enfermeiro $\times$ Paciente & & 4 & 14 & 1 & 19 & 12,3 \\
\hline & Enfermeiro x Grupos comunidade & & & 3 & & 3 & 1,9 \\
\hline & Enfermeiro $x$ Equipe de Saúde & & & 1 & & 1 & 0,6 \\
\hline & Aluno $x$ Pacientes & 1 & & & 1 & 2 & 1,3 \\
\hline Sub.Total & & 2 & 8 & 30 & 4 & 44 & 28,6 \\
\hline \multirow{8}{*}{ Grupos } & Professor $x$ aluno & 1 & 1 & & 1 & 3 & 1,4 \\
\hline & Enfermeiros + alunos & & & & 2 & 2 & 1,3 \\
\hline & Enfermeiros & 4 & 6 & 2 & 10 & 22 & 14,3 \\
\hline & Alunos graduação & 3 & 5 & - & 5 & 13 & 8,4 \\
\hline & Alunos pós-graduação & & & & 1 & 1 & 0,6 \\
\hline & Membros da comunidade/sociedade & 2 & 6 & & 1 & 9 & 5,8 \\
\hline & Equipe de Enfermagem & 4 & 2 & 2 & 2 & 10 & 6,5 \\
\hline & Pacientes/Familiares & 3 & 4 & 10 & 10 & 27 & 17,5 \\
\hline Sub.Total & & 17 & 24 & 14 & 32 & 87 & 56,5 \\
\hline \multirow[t]{2}{*}{ Outros } & $\begin{array}{c}\text { Outros (informações/modelos) } \\
\text { teorias/pesquisas }\end{array}$ & 5 & 6 & 7 & 5 & 23 & 14,9 \\
\hline & $\begin{array}{l}\text { TOTAL } \\
\end{array}$ & 24 & 38 & 51 & 41 & 154 & 100 \\
\hline
\end{tabular}


Pode-se observar 3 categorias de sujeitos: os que evidenciam as interações entre duas pessoas $(28,6 \%)$, com predomínio da díade enfermeiro-paciente(12,3\%), os grupos de sujeitos $(56,5 \%)$ com predomínio de pacientes e familiares $(17,53 \%)$ e enfermeiros $(14,3 \%)$; e outras fontes de informações que são publicações, modelos teóricos, informações administrativas(14,9\%). Nota-se o predomínio de estudos envolvendo tanto o paciente como também profissionais enfermeiros.

Ao se buscar clarificar o objeto dos estudos (Tabela 4) notamos que a maioria se dedica a análise da interação $(45,4 \%)$ seja ela entre profissionais, profissionais-pacientes, profissionais-alunos ou alunospacientes; ou a análise da percepção que um dos elementos envolvidos na interação tem sobre o outro/ outros elementos ou sobre a própria interação(18,2\%).

\section{Tabela 4 - Freqüência dos objetos dos estudos}

\begin{tabular}{|c|c|c|c|c|c|c|}
\hline \multirow{3}{*}{ OBJETO } & \multicolumn{6}{|c|}{ FREQÜENNCLA/ANO } \\
\hline & \multirow[t]{2}{*}{1988} & \multirow[t]{2}{*}{1990} & \multirow[t]{2}{*}{1992} & \multirow[t]{2}{*}{1994} & \multicolumn{2}{|c|}{ Total } \\
\hline & & & & & $\mathrm{N}^{\circ}$ & $\%$ \\
\hline Análise da qualidade da interação & 13 & 16 & 22 & 19 & 70 & 45,4 \\
\hline $\begin{array}{l}\text { Percepção e expectativa de um dos elementos } \\
\text { da interação }\end{array}$ & 4 & 9 & 10 & 5 & 28 & 18,2 \\
\hline $\begin{array}{l}\text { Aspecto da produção/utilização do } \\
\text { conhecimento em Enfermagem }\end{array}$ & 6 & 9 & 08 & 13 & 36 & 23,4 \\
\hline $\begin{array}{llll}\text { Comunicação } & \text { com } & 0 & \text { instrumento } \\
\text { administrativo } & & & \end{array}$ & 0 & 1 & 07 & 4 & 12 & 7,8 \\
\hline $\begin{array}{l}\text { Informações/orientações sobre aspectos da } \\
\text { saúde }\end{array}$ & 1 & 3 & 04 & 0 & 8 & 5,2 \\
\hline TOTAL & 24 & 38 & 51 & 41 & 154 & 100 \\
\hline
\end{tabular}

Por se tratar de uma área de desempenho nem sempre satisfatório, a comunicação tem merecido propostas de alternativas de desenvolvimento de seu processo. Estes estudos caracterizam os aspectos de produção/utilização do conhecimento em Enfermagem $(23,4 \%)$. Nota-se que a comunicação administrativa $(7,8 \%)$ assim como a divulgação $(5,2 \%)$ de informações foram objetos de estudos.

Considerando-se os contextos comunicacionais propostos por LITTLEJOHN(1982), nota-se, pela Tabela 5 , que o contexto interpessoal tem merecido expressiva freqüência nos artigos analisados $(48,1 \%)$. Na literatura, observa-se ao longo do tempo, que as publicações passam por diferentes contextos. Apesar do tema central do $4^{\circ}$ evento contemplar mais especificamente o contexto organizacional, houve ainda predomínio do interpessoal ao longo dos eventos como um todo.

\section{Tabela 5 - Freqüiência dos contextos de comunicação utilizados nos estudos}

\begin{tabular}{l|c|c|c|c|c|c}
\hline \multirow{2}{*}{ CONTEXTO } & \multicolumn{3}{|c|}{ FREQÜENCLA/ANO } & \multicolumn{2}{c}{ TOTAL } \\
\cline { 2 - 7 } & 1988 & 1990 & 1992 & 1994 & $N^{\circ}$ & $\%$ \\
\hline Massa & 3 & 5 & 3 & 4 & 15 & 9,7 \\
\hline Grupal & 8 & 9 & 5 & 5 & 27 & 17,5 \\
\hline Organizacional & 2 & 3 & 3 & 5 & 13 & 8,4 \\
\hline Interpessoal & 10 & 15 & 26 & 23 & 74 & 48,1 \\
\hline Intrapessoal & 1 & 6 & 14 & 4 & 25 & 16,2 \\
\hline TOTAL & $\mathbf{2 4}$ & $\mathbf{3 8}$ & $\mathbf{5 1}$ & $\mathbf{4 1}$ & $\mathbf{1 5 4}$ & $\mathbf{9 9}, 9$ \\
\hline
\end{tabular}

Pode-se dizer que a enfermagem prioriza a compreensão da comunicação na relação enfermeiropaciente. Julgamos ser necessário a realização de investigações sobre a não motivação/condições para estudo da comunicação nos outros contextos.

Em relação a área e ambiente (Tabela 6) notase que a área "Assistência" é a que predomina (40,3\%), em especial no ambiente hospitalar, corroborando com os achados de CARVALHO (1989); ENOKI et al.(1987) e SANDOVAL (1992). No exame da produção científica no Brasil, de 1962 a 1991, BACHION et al.(1992) encontraram também a prevalência da área Assistencial ao longo dos anos, a partir de 1972, sem contudo ser observada a incorporação dos novos conhecimentos à prática. 
Tabela 6 - Freqüência de área e ambiente onde se processam as pesquisas

\begin{tabular}{|c|c|c|c|c|c|c|c|}
\hline AREA & AMBIENTE & \multicolumn{4}{|c|}{ FREQÜENCLA/ANO } & \multicolumn{2}{|c|}{ TOTAL } \\
\hline & & 88 & 90 & 92 & 94 & $\mathrm{~N}^{\circ}$ & $\%$ \\
\hline Administração & $\begin{array}{l}\text { Hospitalar } \\
\text { Comunidade } \\
\text { (não se aplica) }\end{array}$ & $\begin{array}{l}3 \\
0 \\
1\end{array}$ & $\begin{array}{l}4 \\
0 \\
0\end{array}$ & $\begin{array}{l}2 \\
1 \\
0\end{array}$ & $\begin{array}{l}5 \\
0 \\
0\end{array}$ & $\begin{array}{c}14 \\
1 \\
1\end{array}$ & 10,4 \\
\hline Educação & $\begin{array}{l}\text { Escola } \\
\text { Hospitalar } \\
\text { Comunidade }\end{array}$ & $\begin{array}{l}2 \\
0 \\
0\end{array}$ & $\begin{array}{l}5 \\
1 \\
4 \\
\end{array}$ & $\begin{array}{l}1 \\
3 \\
5 \\
\end{array}$ & $\begin{array}{l}8 \\
0 \\
0 \\
\end{array}$ & $\begin{array}{c}16 \\
4 \\
9 \\
\end{array}$ & 18,8 \\
\hline Assistência & $\begin{array}{l}\text { Hospitalar } \\
\text { Ambulatorial } \\
\text { Escola } \\
\text { Empresa } \\
\text { Comunidade }\end{array}$ & $\begin{array}{l}6 \\
- \\
- \\
- \\
-\end{array}$ & $\begin{array}{l}11 \\
- \\
- \\
- \\
-\end{array}$ & $\begin{array}{c}25 \\
3 \\
2 \\
1 \\
1\end{array}$ & $\begin{array}{c}10 \\
1 \\
- \\
- \\
2\end{array}$ & $\begin{array}{c}52 \\
4 \\
2 \\
1 \\
3 \\
\end{array}$ & 40,3 \\
\hline Pesquisa/Conhecimento & $\begin{array}{l}\text { Escola } \\
\text { Hospital } \\
\text { (não se aplica) }\end{array}$ & $\begin{array}{l}3 \\
2 \\
7\end{array}$ & $\begin{array}{l}2 \\
6 \\
5\end{array}$ & $\begin{array}{l}1 \\
3 \\
3 \\
\end{array}$ & $\begin{array}{l}4 \\
2 \\
9\end{array}$ & $\begin{array}{l}10 \\
13 \\
24 \\
\end{array}$ & 30,5 \\
\hline TOTAL & & 24 & 38 & 51 & 41 & 154 & 100 \\
\hline
\end{tabular}

Nota-se na área "Pesquisa/Conhecimento" $(30,5 \%)$ a preocupação com a produção de conhecimento alicerçado em novas propostas ou análise do conhecimento já produzido, evidenciada pela categoria "não se aplica" quanto a ambiente. Os ambientes da escola e do hospital também surgem como locais de produção de conhecimento. A área "Educação" $(18,8 \%)$ centraliza seus estudos nos ambientes escolares, da comunidade. Isto nos faz crer que o processo educativo dos pacientes internados não esteja ocorrendo de forma destacada como tal, mas talvez de forma mesclada, como processo inerente à relação enfermeiro-paciente.

A área menos pesquisada foi "Administração" $(10,4 \%)$ e as pesquisas aqui arroladas privilegiaram o ambiente hospitalar. Como indica LITTLEJOHN(1982), a comunicação permeia a administração, assim, estudar a comunicação nesse contexto seria estudar a própria administração.

Quanto ao tipo de pesquisa, (Tabela 7) outra variável estudada, observamos que na maioria não há interferência ou controle por parte do pesquisador; são estudos não experimentais( $89,6 \%)$, em especial descritivos. É ainda incipiente a freqüência da manipulação na pesquisa por parte do experimentador, seja ela parcial $(5,8 \%)$ ou completa $(4,5 \%)$. Este fato pode evidenciar que a natureza do objeto é de dificil manipulação ou refletir estarem os pesquisadores em fase inicial da produção do conhecimento nesta área, como já destacaram anteriormente BACHION \& CARVALHO(1992).

Tabela 7 - Freqüência dos tipos de pesquisa utilizados nos estudos

\begin{tabular}{|c|c|c|c|c|c|c|}
\hline \multirow[t]{2}{*}{ TIPO PESQUISA } & \multicolumn{4}{|c|}{ FREQÜÊNCIA/ANO } & \multicolumn{2}{|c|}{ TOTAL } \\
\hline & 1988 & 1990 & 1992 & 1994 & $\mathrm{~N}^{\circ}$ & $\%$ \\
\hline $\begin{array}{r}\text { Não Experimental - Descritiva } \\
\text { - Pós Facto }\end{array}$ & $\begin{array}{c}19 \\
3\end{array}$ & $\begin{array}{l}26 \\
11\end{array}$ & $\begin{array}{l}34 \\
8\end{array}$ & $\begin{array}{c}36 \\
1\end{array}$ & $\begin{array}{c}115 \\
23\end{array}$ & $\begin{array}{l}74,7 \\
14,9\end{array}$ \\
\hline Quase Experimento & - & - & 9 & - & 9 & 5,8 \\
\hline Experimento & 2 & 1 & 0 & 4 & 7 & 4,5 \\
\hline TOTAL & 24 & 38 & 51 & 41 & 154 & 100 \\
\hline
\end{tabular}

Anteriormente, CARVALHO (1989), em ampla revisão, já constatou o pequeno número de estudos experimentais, acerca da Comunicação em Enfermagem, a nível nacional. Isso reforça nossa percepção de que estamos em fase inicial da abordagem desse assunto, tão extenso e complexo.

Quanto aos quase experimentos, são estudos onde os autores atuavam de modo premeditado na interação, 
caracterizando, para nós, um tipo de controle, isto é, o controle do que era falado ou da forma de abordagem, conscientemente planejada para ser terapêutica. Acreditamos que estudos dessa natureza em muito colaboram no desenvolvimento de corpo de conhecimento, já que favorecem a auto-reflexão e estimulam a busca de novas estratégias de abordagem, e acima de tudo, possibilitam a melhoria da assistência ao paciente, que tem, de um modo mais claro e efetivo, suas necessidades atendidas ou problemas atenuados e permite ao profissional conhecer mais profundamente a clientela objeto de seu trabalho.

Outro ponto que, em nosso entender, traz contribuições para o fortalecimento das bases do exercício profissional é a utilização/validação/desenvolvimento de referenciais teóricos. Das pesquisas publicadas, a maioria fundamentou explicitamente em referenciais de Comunicação, tanto em obras específicas do assunto como obras gerais que o contemplam como tópico.

Sobressaem os pressupostos de BALES (1950); SULLIVAN (1953 a, 1953b); BERLO (1960); RUESCH(1964); WATZLAWIC et al. (1981); LITTLEJOHN(1982); SKINNER(1957); WEISS(1979); BELTRÃO(1982); KNAPP(1985); ROGERS(1991); DAVIS(1979); RECTOR \& TRINTA (1985); BORDENAVE(1987); MEHRABIAN(1971); Hall apud LITTLEJOHN, (1982); McLUHAN(1984) e a aplicação dos preceitos de comunicação em enfermagem, como os de PEPLAU(1952); LEWIS(1973); VIEIRA(1978); FORREST (1983); DUGAS (1984); KING et al. (1981); TRAVELBEE (1982); DANIEL (1983); STEFANELLI (1985); VIETTA(1985); MENDES(1986); RODRIGUES (1986); MANZOLLI (1987); SAWADA (1990); BONADIO et al. (1990), além das considerações de CARVALHO $(1985,1989)$ sobre comunicação como processo.

Estes achados contrapõem-se aos anteriormente citados na literatura nacional, que apontam o pequeno uso de modelos ou referenciais teóricos nos estudos realizados e corroboram a percepção de BACHION(1994), de que o desenvolvimento de tal área vem ocorrendo dando cada vez mais subsídios aos pesquisadores e docentes de enfermagem.

Ao analisar os achados da produção examinada, buscando compor o panorama do estado da comunicação na assistência, no ensino e por último, na pesquisa de enfermagem, podemos considerar que:

- na instância assistencial os achados da produção analisada nos apontam fatos que impõe reflexão. Evidenciam que as orientações à clientela não são adequadas nem eficientes. Mesmo quando está fisicamente perto da clientela, a enfermagem não estabelece com ela uma relação de boa qualidade. As interações estão centradas na tarefa, são muitas vezes superficiais, impessoais e não obedecem a um contínum de começo, meio e fim. Por outro lado também é apontado que uma intervenção mais pessoal é possível, desde que haja autoconsciência e exercício de reflexão sobre as próprias ações e as do outro. Educação continuada neste sentido poderia favorecer o aprimoramento profissional nessa área.

- a dimensão da administração no ambiente assistencial ainda não se constitui objeto marcante das pesquisas de comunicação, as que ocorrem evidenciam ainda a inadequacidade de anotações de enfermagem, as dificuldades da passagem de plantão, fatos estes conhecidos de todos nós de longa data. Demonstra-se também o sucesso na implantação de um instrumento de comunicação administrativa.

- na instância da educação/ensino, a situação é também delicada. Podemos pensar na comunicação enquanto estratégia ou como conteúdo a ser desenvolvido. É do nosso conhecimento vários estudos que se ocuparam dessa temática como os de CANCHE et al.(1988); SONOBE(1990); CARVALHO et al.(1988); FERRAZ et al.(1988); ROLIM (1981), que retratam a situação do ensino de comunicação e outros, como o de CARVALHO(1985); STEFANELLI(1985) e BACHION(1994) que se preocupam em estabelecer propostas para este ensino. Vale ressaltar que este tópico constou de mesa redonda no III SIBRACEn, sendo focalizados tanto o ensino a nível de graduação como o de pós-graduação;

- quanto às investigações que revelam o estado da pesquisa em Comunicação em Enfermagem, temos que está sendo mais usual a aceitação de instrumento para registro de dados. Em nosso entender, a partir do momento em que a aparelhagem de audio e vídeo se tornarem mais familiares para os pesquisadores e "pesquisados", se instalará um clima de naturalidade frente ao registro, favorecendo o procedimento da investigação.

Ainda, no tocante à área de pesquisa, os trabalhos evidenciam a discussão sobre a concepção dos teoristas de Enfermagem e dos enfermeiros acerca da comunicação. Empreendeu-se esforços no sentido de caracterizar o perfil dos pesquisadores e as tendências de suas investigações. Os achados, de modo geral, são que: os aspectos do processo de comunicação que permeiam as teorias estão ligados às diferentes concepções do homem e da enfermagem; o conceito de comunicação nas pesquisas aparece relacionado a uma série de outros conceitos (o que para nós reforça o caráter de multifacetariedade deste tema); geralmente se enfoca a comunicação do ponto de vista da interação verbal: sua importância é atribuida no âmbito da assistência direta; finalmente, a percepção da adequacidade das investigações realizadas e de sua aplicabilidade não é uniformemente aceita entre os enfermeiros assistenciais. 


\section{CONCLUSÃO}

Reportando-nos aos objetivos propostos para este estudo, podemos assim respondê-los:

- os pesquisadores que realizam trabalhos na área da Comunicação em Enfermagem são na maioria enfermeiros docentes, que operam em grupos para investigar díades - profissionais e clientela, no tocante ao desempenho dos primeiros e ainda a percepção desta em relação a situações vivenciadas, principalmente na área da assistência hospitalar, configurando estudos descritivos, alicerçados em referenciais teóricos interacionistas;

- em termos gerais os resultados apontam para a inadequacidade da situação nos vários âmbitos da comunicação, porém, ao mesmo tempo, configura-se a busca de soluções criativas e preocupação com testes de novas alternativas (ao buscar produzir novos conhecimentos nesta área) e do avanço da produção científica de comunicação em enfermagem.

\section{RETROSPECTIVE ANALYSIS OF THE SCIENTIFIC PRODUCTION PRESENTED AT THE BRAZILIAN SYMPOSIUM ON NURSING COMMUNICATION - SIBRACEn: (1988-1994)}

The present study had the purpose of identifying characteristics of the scientific production on Nursing Communication based on a sample of papers presented at four events of the Brazilian Symposium on Nursing Communication (1988, 1990, 1992 and 1994). The majority of authors were faculty, who developed research mainly in groups, focussing as subjects the dyad (professionals and clientele), establishing as objects of their studies aspects of interaction (quality and effectiveness) as well as aspects of the production and utilization of Nursing knowledge. Interpersonal context was the most investigated, care was the area most studied and the usual environment was the hospital. Research were mainly of non-experimental type. Results evidenced aspects of communication inadequateness in areas of care (including administration), education (mainly of clientele) and research. At the same time, authors perceived the trend towards innovative solutions and improvement of knowledge on the communication process.

KEY WORDS: nursing communication, scientific production

\section{RETROSPECTIVA DE LA PRODUCCIÓN CIENTÍFICA DEL SIBRACEn: (1988-1994)}

En este estudio se buscó identificar algunas características de la producción científica sobre Comunicación en Enfermería tenendo en consideración los trabajos presentados en los cuatro eventos del Simpósio Brasileño de Comunicación en Enfermería (1988, 1990, 1992 y 1994). Se verificó que la mayoría de los autores está compuesta por docentes, que realizan sus investigaciones en grupos, enfocando principalmente como sujetos las diades (profesionales y clientela), estabeleciendo como objeto de estudio aspectos de la interacción (cualidad y efectividad) y aspectos de la producción/utilización del conocimiento en Enfermería. El contexto interpersonal es lo más investigado. El área más estudiada es la asistencial y el ambiente es el hospital. La mayoría de las investigaciones son del tipo no experimento. Los resultados evidenciaron aspectos de inadecuacidad de la comunicación en área de asistencia (incluyendo la administración), educación (principalmente la clientela) e investigación. Al mismo tiempo, percíbese una tendencia de búsqueda por soluciones innovadoras, también por el avance del conocimento sobre el proceso de comunicación.

TÉRMINOS CLAVES: comunicación en enfermería, producción cientifica

\section{REFERÊNCIAS BIBLIOGRÁFICAS}

\section{AGUIAR, M.G.G. et al Análise da pesquisa na área} de comunicação em enfermagem no período compreendido entre 1985 a 1990. Curitiba, 1991. (mimeografado) /Apresentado no $43^{\circ}$ Congresso Brasileiro de Enfermagem/.

02. BACHION, M.M. Comunicação interpessoal: ensino de algumas habilidades desejadas. Ribeirão Preto, 1994. Tese (Doutorado) - Escola de Enfermagem de Ribeirão Preto, Universidade de São Paulo.
03. BACHION, M.M.; CARVALHO, E.C. Pesquisa e pesquisadores da comunicação em enfermagem. In: ENCONTRO INTERNACIONAL PESQUISA DE ENFERMAGEM: UMA QUESTÃO DE SAÚDE, 1, São Paulo, 24 a 30 de outubro de 1992. Programa. São Paulo: Escola de Enfermagem da USP - 1992.

04. BACHION, M.M.; CHIANCA, T.C.M.; MALUEZZI, G.M.; JORGE, M.S. Um estudo sobre a produção da pós-graduação de enfermagem no Brasil, no período de 1962 a 1991. In: ENCONTRO INTERNACIONAL PESQUISA EM ENFERMAGEM: UMA QUESTÃO DE SAÚDE, 1, São Paulo, 24 a 20 de outubro de 1992. Programa. São Paulo: Escola de Enfermagem - USP, 1992. p. 143/Resumo. 
05. BALES, R.F. Interaction process analysis: a method for the study of small groups. Cambridge: Addison - Wesley Press, 1950.

06. BELTRÃO, L. Teoria geral de comunicação. 3. ed. Brasília: Thesaurus, 1982.

07. BERLO, D.K. O processo de comunicação. Rio de Janeiro: Fundo de Cultura, 1960.

08. BONADIO, I.C.; OLIVEIRA, S.M.J.V.; YOSHIOCA, M.R. A Comunicação na prática dos profissionais de saúde. In: SIMPÓSIO BRASILEIRO DE COMUNICAÇÃO EM ENFERMAGEM, 2, Ribeirão Preto, 2 - 4 maio de 1990. Anais. Ribeirão Preto: Escola de Enfermagem de Ribeirão Preto - USP, 1990. p. 539-551.

09. BORDENAVE, J.E.D. - O que é comunicação? 10.ed. São Paulo: Brasiliense, 1987.

10. CANCHE, R.F.; SONOBE, H.M.; BERTAZONE, E.C. A comunicação aluno/paciente segundo a percepção de graduandos de enfermagem. In: SIMPÓSIO BRASILEIRO DE COMUNICAÇÃO EM ENFERMAGEM, 1, Ribeirão Preto, 2 - 4 maio. 1988. Anais. Ribeirão Preto: Escola de Enfermagem de Ribeirão Preto - USP, 1988. p. 175-187.

11. CARVALHO, E.C. Comportamento verbal enfermeira-paciente: função educativa e educação continuada do profissional. Ribeirão Preto, 1985. 225p. Tese (Doutorado) - Escola de Enfermagem de Ribeirão Preto, Universidade de São Paulo.

12. CARVALHO, E.C. Enfermagem e comunicação: a interface. Ribeirão Preto, 1989. 245p. Tese (Livre-docência) - Escola de Enfermagem de Ribeirão Preto, Universidade de São Paulo.

13. CARVALHO, E.C. Os contextos de comunicação em enfermagem. In: SIMPÓSIO BRASILEIRO DE COMUNICAÇÃO EM ENFERMAGEM, 2, Ribeirão Preto, 2 a 4 maio, 1990. Anais. Ribeirão Preto: Escola de Enfermagem de Ribeirão PretoUSP, 1990. p. 2-23.

14. CARVALHO, E.C.; FERRAZ, A.E.P.; GIR, E. O ensino de comunicação nas Escolas de Enfermagem do Brasil. In: SIMPÓSIO BRASILEIRO DE COMUNICAÇÃO EM ENFERMAGEM, 1, Ribeirão Preto, 2 - 4 maio, 1988. Anais. Ribeirão Preto: Escola de Enfermagem de Ribeirão Preto - USP, 1988. p. 96-108.

15. DANIEL, L.F. Atitudes interpessoais em enfermagem. São Paulo: EPU, 1983.

16. DAVIS, P. A comunicação não verbal. Trad. de Antônio Dimas. São Paulo: Summus, 1979.

17. DUGAS, B.W. Enfermagem prática. 4. ed. Rio de Janeiro: Interamericana, 1984.
18. ENOKI, H.; FERRAZ, E.P.; CARVALHO, E.C.; MARZIALE, M.H.P. A produção científica acerca da comunicação em enfermagem. Rev. Bras. Enfermagem, v.40, n.1, p.34-7, 1987.

19. FERRAZ, B.W.; MARZIALE, M.H.P.; GIR, E.; CARVALHO, E.C.; MORIYA, T.M. O processo de comunicação em enfermagem: visão do aluno de graduação. In: SIMPÓSIO BRASILEIRO DE COMUNICAÇÃO EM ENFERMAGEM, 1. Ribeirão Preto, 2 - 4 maio 1988. Anais. Ribeirão Preto: Escola de Enfermagem de Ribeirão Preto - USP, 1988. p. 162-174.

20. FORREST, D. Analysis of nurses verbal communication with patients. Nur. Pap., v.15, n.3, p.48-57, 1983.

21. KING, D.; BARNARD, K.E.;HOEHN, R. Dissemanating the results of nursing research. Nurs.Outlook, v.29, n.3, p.164-69, 1981.

22. KNAPP, M.L. La comunicación no verbal: el cuerpo y el entorno. Barcelona: Paidós, 1985.

23. LEWIS, G.K. Nurse-patient communication. Dubuque: W.N.C. Brown, 1973.

24. LITTLEJOHN, S.W. Fundamentos teóricos da comunicação. Trad. de A. Cabral. Rio de Janeiro: Zahar, 1982.

25. MANZOLLI, M.C. Relacionamento em enfermagem. 2. ed. São Paulo: Sarvier, 1987.

26. McLUHAN, M. Os meios de comunicação como extensões do homem. São Paulo: Cultrix, 1984.

27. MEHRABIAN, A. Silent messages. Belmont: Wadsworth, 1971.

28. MENDES, I.A.C. Interação verbal em situações de enfermagem hospitalar: enfoque humanistico. Ribeirão Preto, 1986. Tese (Doutorado) - Escola de Enfermagem de Ribeirão Preto, Universidade de São Paulo.

29. PEPLAU, H.E. Interpersonal relations in nursing. New York: Putnam's, 1952.

30. POLIT, D.F.; HUNGLER, B.P. Nursing research: principles and methods. 4th ed. Phyladelphia: J.B. Lippincott, 1991. 697p.

31. RECTOR, M.; TRINTA, A.R. Comunicação nãoverbal: a gestualidade brasileira. Petrópolis: Vozes, 1985.

32. RODRIGUES, A.R.F. Enfermagem de saúde mental para mulheres em crise acidental. Ribeirão Preto, 1986. Tese (Doutorado) - Escola de Enfermagem de Ribeirão Preto, Universidade de São Paulo.

33. ROGERS, C. Tornar-se pessoa: psicologia e pedagogia. São Paulo: Martins Fontes, 1991.

34. ROLIM, M.A. A utilização de padrões não terapêuticos de comunicação na interação alunopaciente. Rev.Esc. Enfermagem USP, v. 15, n.2. p. 173-177, 1981. 
35. RUESCH, J. Comunicación terapeutica. Buenos Aires: Paidós, 1964.

36. SANDOVAL, J.M.H. Um estudo sobre estudos de comunicação em enfermagem. In: SIMPÓSIO BRASILEIRO DE COMUNICAÇÃO EM ENFERMAGEM, 3, Ribeirão Preto, 13-14 maio 1992. Anais. Ribeirão Preto: Escola de Enfermagem de Ribeirão Preto-USP, 1992. p. 646-600.

37. SAWADA, N.O. A dimensão não verbal da interação enfermeiro-paciente em situação pré-operatória. Ribeirão Preto, 1990. Dissertação (Mestrado) - Escola de Enfermagem de Ribeirão Preto, Universidade de São Paulo.

38. SKINNER, B.F. Verbal behavior. NewYork: Appleton Century Crofts, 1957.

39. SONOBE, H.M. Comunicação aluno-paciente: questões e obstáculos. In: SIMPÓSIO BRASILEIRO DE COMUNICAÇÃO EM ENFERMAGEM, II, Ribeirão Preto, 2 - 4 maio 1990. Anais. Ribeirão Preto: Escola de Enfermagem de Ribeirão Preto-USP, 1990. p. 552-565.
40. STEFANELLI, M.C. Ensino de técnicas de comunicação terapêutica enfermeira-paciente. São Paulo, 1985. 163p. Tese (Doutorado) - Escola de Enfermagem, Universidade de São Paulo.

41. SULLIVAN, H.S. The interpersonal theory of psychiatry. NewYork: Norton, 1953a.

42. Conceptions of modern psychiatry. NewYork: Norton 1953b.

43. TRAVELBEE, J. Intervencíon en enfermería psiquiatrica. 2. ed. Washington: OPS-OMS, 1982.

44. VIEIRA, T.T. O processo da comunicação na enfermagem. Salvador: UFBa - Centro Editorial, 1978.

45. VIETTA, E.P. Marco conceitual para a prática de enfermagem social: contribuição para bases de uma teoria de enfermagem. Ribeirão Preto, 1985. Tese (Doutorado) - Escola de Enfermagem de Ribeirão Preto, Universidade de São Paulo.

46. WATZLAWIC, P. et al. Pragmática da comunicação humana. São Paulo: Cultrix, 1981.

47. WEISS, S.J. The language of touch. Nurs.Res., v.28, n.2, p.76-80, Mar/Apr. 1979. 